\title{
Effects of prefrontal tDCS on executive function: Methodological considerations revealed by meta-analysis
}

\author{
Michael J. Imburgio ${ }^{\mathrm{a}, *}$, Joseph M. Orr ${ }^{\mathrm{a}, \mathrm{b}, * *}$ \\ a Department of Psychological and Brain Sciences, Texas A\&M University, College Station, TX, USA \\ ${ }^{\mathrm{b}}$ Texas A\&M Institute for Neuroscience, Texas A\&M University, College Station, TX, USA
}

\section{A R T I C L E I N F O}

\section{Keywords:}

TDCS

Executive Function

DLPFC

Meta-analysis

\begin{abstract}
A B S T R A C T
A meta-analysis of studies using single-session transcranial direct current stimulation (tDCS) to target the dorsolateral prefrontal cortex (DLPFC) was undertaken to examine the effect of stimulation on executive function (EF) in healthy samples. 27 studies were included in analyses, yielding 71 effect sizes. The most relevant measure for each task was determined a priori and used to calculate Hedge's g. Methodological characteristics of each study were examined individually as potential moderators of effect size. Stimulation effects on three domains of EF (inhibition of prepotent responses, mental set shifting, and information updating and monitoring) were analyzed separately. In line with previous work, the current study found no significant effect of anodal unilateral tDCS, cathodal unilateral tDCS, or bilateral tDCS on EF. Further moderator and subgroup analyses were only carried out for anodal unilateral montages due to the small number of studies using other montages. Subgroup analyses revealed a significant effect of anodal unilateral tDCS on updating tasks, but not on inhibition or setshifting tasks. Cathode location significantly moderated the effect of anodal unilateral tDCS. Extracranial cathodes yielded a significant effect on EF while cranial cathodes yielded no effect. Anode size also significantly moderated effect of anodal unilateral tDCS, with smaller anodes being more effective than larger anodes. In summary, anodal DLPFC stimulation is more effective at improving updating ability than inhibition and setshifting ability, but anodal stimulation can significantly improve general executive function when extracranial cathodes or small anodes are used. Future meta-analyses may examine how stimulation's effects on specific behavioral tasks, rather than broader domains, might be affected by methodological moderators.
\end{abstract}

\section{Introduction}

Higher order control over behavior, known as executive function, is a necessary component of every day functioning. Executive function underlies our ability to manipulate information to form plans, remember how to follow a recipe, and switch from reading an email to reading the news. Executive function is widely considered to be a function of the prefrontal cortex. Neuroimaging work has informed theories that depict the dorsolateral prefrontal cortex (DLPFC) as central to executive functioning (Miller and Cohen, 2001). Indeed, damage to the DLPFC leads to executive function deficits such as the inability to follow schedules and initiate actions (Ptak and Schnider, 2004).

However, executive function is a diverse construct and the involvement of the DLPFC in every aspect of executive function may not be uniform. The Unity-Diversity model (Miyake et al., 2000) posits that executive function is composed of inhibition, set-shifting and updating components. The inhibition domain of executive function is characterized by tasks requiring inhibition of prepotent responses, such as a Stroop task. Set-shifting is captured primarily by task switching paradigms and involves flexibly adapting to a relevant task and disengaging from an irrelevant task. Updating involves dynamic manipulation of working memory contents and is measured by paradigms such as the n-back. It is possible that while the DLPFC is active during all three domains of executive function, it is integral to some domains and peripheral to others. For example, while a review found consistent support for crucial involvement of the DLPFC in working memory (Curtis and D'Esposito, 2003), similar reviews and meta-analyses have found less convincing evidence for the DLPFC's centrality to inhibition and setshifting (Aron et al., 2004; Wager et al., 2004). Non-invasive stimulation techniques that alter DLPFC functioning may be used to determine the magnitude of DLPFC involvement in each of these domains by examining the degree to which the domain is affected behaviorally.

Transcranial direct current stimulation (tDCS) attempts to modulate neural firing in a targeted area by passing a small amount of current

\footnotetext{
* Correspondence to: 515 Coke Street, 4235 TAMU, College Station, TX 77843-4235, USA.

** Corresponding auther.

E-mail addresses: mimburgi@tamu.edu (M.J. Imburgio), joseph.orr@tamu.edu (J.M. Orr).
} 
through two (or more) electrodes placed on the scalp. tDCS can be used to either upregulate or downregulate brain activity depending on the direction of the current flow. Anodal tDCS (a-tDCS) is generally considered excitatory while cathodal tDCS (c-tDCS) is generally considered inhibitory. However, studies examining the effect of motor cortex stimulation have revealed that individual responses to stimulation are not uniformly excitatory or inhibitory. Baseline performance, baseline neurotransmitter levels, genetics and anatomical factors can affect the degree and direction of the effect of stimulation (Li et al., 2015). Furthermore, there is always both cathodal and anodal stimulation in a given paradigm; every stimulation montage includes at least one anode and at last one cathode. Additionally, stimulation might act via neurotransmitter modulation (Antonenko et al., 2017; Bachtiar et al., 2015; Stagg and Nitsche, 2011). However, the relationship between neurotransmitter levels and executive function performance is not linear (Cools and D'Esposito, 2011; Robbins and Arnsten, 2009). Therefore, anodal stimulation may not uniformly improve executive function and cathodal stimulation may not uniformly worsen executive function. Indeed, recent meta-analyses examining the effectiveness of tDCS on cognition in general concluded there was no significant effects of stimulation in healthy populations (Dedoncker et al., 2016; Horvath et al., 2015). While these meta-analyses demonstrate a lack of overall stimulation effects by examining between-study variability, within-study variability resulting from individual differences in stimulation reactivity might underlie the null effects found in these analyses.

Between-study variability has also been demonstrated in the above analyses. Between-study variability may result from methodological differences among studies; stimulation intensities, reference electrode placements, and stimulation durations often vary greatly. Such factors can have significant effects on tDCS efficacy in general, although they have not been explored specifically with respect to executive function (Dedoncker et al., 2016; Tremblay et al., 2014). Indeed, while previous analyses have found no effect of tDCS on executive function (Horvath et al., 2015) and no effect of DLPFC tDCS on general cognition (Dedoncker et al., 2016), the effect of DLPFC tDCS on executive function specifically has not been explored. Furthermore, no previous analyses have addressed how the effects of stimulation may differ with regard to executive function domain.

Understanding the effects of DLPFC stimulation on individual domains of executive function in healthy individuals might provide a lens through which stimulation as treatment method can be understood. There is evidence that a-tDCS of the DLPFC may be an effective treatment method for major depressive disorder (MDD) and schizophrenia (Boggio et al., 2008; Brunelin et al., 2012; Brunoni et al., 2011; Fregni et al., 2006). Reviews of people with MDD and schizophrenia have found deficiencies in executive function associated with abnormal DLPFC activity (Fossati et al., 2002; Minzenberg et al., 2009; Rogers et al., 2004), and treatment resulting in a reduction of symptoms in is often accompanied by improvements in executive function (Biringer et al., 2005; Meltzer and McGurk, 1999; Walter et al., 2010). As such, it is possible that stimulation of the DLPFC results in symptom improvement in people with MDD and schizophrenia by improving executive function. Understanding the cognitive mechanism behind possible executive function improvements caused by stimulation in healthy individuals will aid our understanding of the mechanism of treatment in these disoders.

However, the efficacy of DLPFC tDCS as a treatment method is inconsistent. Recent meta-analyses have generally concluded that tDCS is an effective treatment for MDD (Kalu et al., 2012; Shiozawa et al., 2014), but disagreement exists in the literature (Berlim et al., 2013). As is the case in healthy participants, inter-subject variability in responsiveness likely plays a role in these inconsistencies, as does variations in the methods applied during treatment. Understanding the effects of methodological choices such as reference electrode placement and stimulation intensity on healthy individuals may shed light on these inconsistencies in treatment effectiveness.
The current work aims to understand the effects of DLPFC stimulation on global executive function and the individual domains of executive function defined by the Unity-Diversity model. Quantifying the effects of stimulation on domains of executive function rather than simply global executive function will provide a more nuanced understanding of DLPFC involvement in behavior. Additionally, the moderating effects of methodological factors on stimulation efficacy are examined. Understanding how factors such as stimulation intensity, stimulation duration, and reference electrode placement influence executive function during DLPFC stimulation is a significant step towards establishing standard practice in the field that might limit betweenstudy variability in results.

\section{Material and methods}

\subsection{Study selection and qualitative coding}

Articles were obtained from searches of PubMed, ScienceDirect, Web of Science, DART, and ProQuest (3/14/2017). Search terms were (1) "DLPFC" OR "Dorsolateral prefrontal cortex", AND (2) "tDCS" OR "transcranial direct current stimulation" AND (3) "executive function" OR "inhibition" OR "cognitive control" OR "cognitive flexibility" OR "working memory." Duplicates between databases and review articles were removed. Trained research assistants reviewed search results to confirm that the study included a healthy population, applied tDCS to the DLPFC, included a cognitive outcome measure, and included a sham tDCS group. Studies were removed if they attempted to target the DLPFC but placed the DLPFC electrode somewhere other than F3 or F4. Studies were only included if they included one active tDCS session. Performing a task during stimulation may alter the stimulation effects on related tasks post-stimulation (Gill et al., 2015; Ruf et al., 2017). If a cognitive task was performed during stimulation and a related cognitive task after stimulation, only the task performed during stimulation was considered for analysis. Additionally, an a priori decision was made to exclude studies which did not include at least a three hour period between sham stimulation and active stimulation conditions to account for any carryover effects of stimulation.

A subsample of 20 of the remaining studies were then double coded by M.I. and J.O. to determine whether the cognitive task in each paper fell under an executive function domain as defined by Miyake et al. (2000). To account for situations in which a low difficulty version of a task may not fall under one of the three domains, but a higher difficulty would, only the most difficult version of a task in a given study were analyzed. For example, a Sternberg task with one target letter may only require simple recognition and recall while a Sternberg task with 5 target letters may require continuous manipulation and updating of working memory, thus only Sternberg performance on 5-letter trials were included. Additionally, if the task was determined to fall under an executive function domain, the outcome measure most relevant to the domain (for example, RT Stroop Effect for the Stroop task) was double coded. Inter-rater reliability was 0.85 . Discrepancies were resolved by consensus. The remaining studies were then coded for domain.

The remaining studies were then coded for the following qualitative information: sample size, gender composition, sample age, electrode placement, electrode size, stimulation intensity, stimulation duration, stimulation direction, stimulation timing (online, offline or a combination), and stimulation laterality. All qualitative variables coded were assessed as potential moderators. Moderators were chosen based on previous reviews and analyses that examined the effects of tDCS (Dedoncker et al., 2016; Horvath et al., 2015; Tremblay et al., 2014).

Because we were interested specifically in effects of DLPFC stimulation, polarity and laterality were coded only with respect to the F3/F4 sites; if the anode electrode was placed over F3 and the cathode electrode was not placed over F4, the stimulation was considered anodal and unilateral. Because in this example only the left DLPFC would be targeted, such a montage is nonetheless considered anodal and 
unilateral. Similarly, a study was only considered bilateral if it specifically targeted both left and right DLPFC (both F3 and F4), despite the fact that some studies targeting only F3 or F4 likely include incidental stimulation of the other site. Incidental stimulation to opposite areas is assessed by examining the moderating effects of reference electrode location. This terminology is consistent with usage in the extant literature. Note, however, that these terms are very often used incorrectly, or at the very least, are misleading. Positive stimulation over F3 does not necessarily mean that the current is restricted to the left DLPFC area; as demonstrated in Fig. 5, there is often a wide area of stimulation, especially with traditional 2-channel cranial electrode stimulation. Additionally, many studies included multiple executive function-related tasks or multiple stimulation parameters. In these cases, effect sizes for each task and stimulation parameter are considered separately. As such, many studies yielded multiple effect sizes.

\subsection{Quantitative analyses}

All statistical analyses were performed in R v3.1.1 (R Core Team, 2016). Hedges' $g$ was computed for each study using mean differences and pooled standard deviation. Hedges' $g$ was preferred over Cohen's d due to possible bias in Cohen's d during subgroup analyses in which the number of analyzed effect sizes is small (Hedges, 1981). If means and standard deviations for the relevant metric were not reported but were graphed, the values were extracted using WebPlotDigitizer 3.12 (Rohatgi, 2017). Difference scores between pre- and post-stimulation performance were used when available. In a small amount of cases $(N$ $=2$ ), means and standard deviations for the relevant task were not reported nor graphed and were not able to be obtained from authors, thus Hedges' $g$ was calculated using reported t values. Additionally, in a small amount of cases $(N=2)$, the previously defined most relevant measure (Stroop Effect) was not reported nor graphed, and authors did not reply to requests for data; Thus, pre- and post-stimulation incongruent trial RT was used. Anodal and cathodal stimulation scores were then compared individually to sham stimulation scores. Bilateral was considered separately from unilateral stimulation effects. Direction of effect size was corrected such that a positive effect size indicates improved cognitive control (e.g., reduced switch cost) and a negative effect size indicates decreased cognitive control (e.g., increased switch cost) relative to sham.

The Chi-square test was used to assess heterogeneity of effect sizes. A DerSimonian-Laird (DL) mixed-effects model was used for all analyses to account for expected heterogeneity caused by methodological differences. Meta-regression analyses were carried out for each potential qualitative moderator separately. Meta-regression analyses of categorical moderators were only performed if there were at least 10 effect sizes in each category of the moderator. If meta-regression analyses yielded at least a trending moderator, subgroup analyses were performed to examine whether the effect size of each group significantly differed from zero.

\section{Results}

The original searches of PubMed, ScienceDirect, Web of Science, ProQuest, and DART yielded 2460 results. After removal of duplicates, studies that did not target F3 or F4, training studies, studies that did not include a healthy population, studies that did not target a behavior related to higher-level cognition, and reviews, 55 studies remained. See Fig. 1 for a breakdown of study inclusion. After eliminating studies for which none of the tasks used were consistent with the definition of executive function put forth by Miyake et al. (2000), most of which were related to emotion regulation or risk preference, 29 studies remained. Of the 29 remaining studies, effect sizes were not able to be computed from 2 due to missing data that could not be obtained from authors, leaving 27 studies to be analyzed. Due to the fact many studies contained multiple stimulation parameters and/or multiple tasks, the

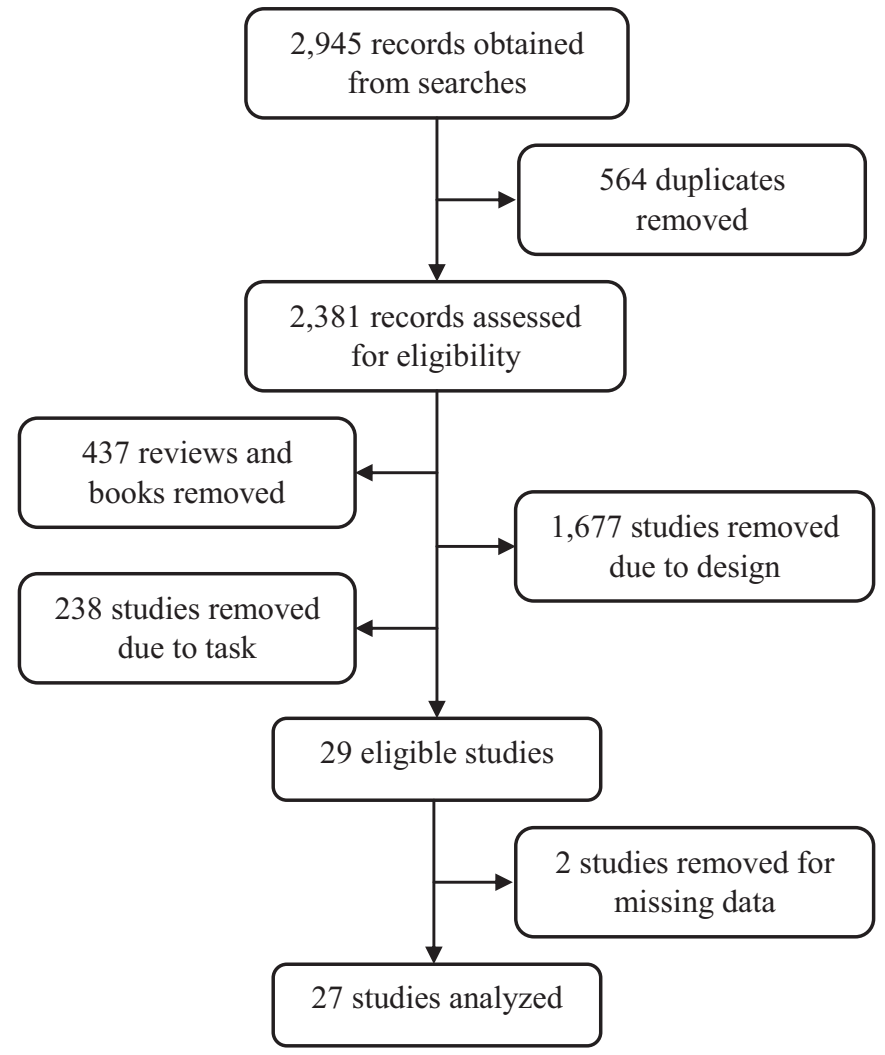

Figure 1. Graphical depiction of study inclusion procedure.

27 studies yielded 71 effect sizes (see Table 1 and Mendeley Data set).

\subsection{Main effect of stimulation type on general executive function}

We first examined the effects of tDCS across the Miyake et al. domains of executive function. Analysis of unilateral a-tDCS effects on executive function did not yield a significant effect size, $g=0.03$ (0.14), $p=0.82$ (see Fig. 2A). The Chi-square test for heterogeneity was significant, $I^{2}=51.04 \%, Q(37)=75.57, p<0.001$. Analysis of unilateral c-tDCS effects on executive function did not yield a significant effect size, $g=-0.05(0.13), p=0.65$. The Chi-square test for heterogeneity was not significant, $I^{2}=0 \%, Q(13)=11.83, p=0.89$. Analysis of bilateral tDCS effects on executive function did not yield a significant effect size, $g=0.05$ (0.17), $p=0.75$. The Chi-square test for heterogeneity was not significant, $I^{2}=0 \%, Q(12)=6.26, p=0.9$. The subsequent moderator and subgroup analyses were constrained to unilateral a-tDCS because (1) neither the effects of unilateral c-tDCS nor bilateral tDCS were found to be significantly heterogenous and (2) the large number of unilateral a-tDCS effect sizes was amenable to the examination of most potential moderators.

\subsection{Subgroup analysis of executive function domain}

We then turned to the individual domains of executive function as outlined by Miyake et al. (2000), shifting, updating, and inhibition. Analysis of unilateral a-tDCS effects on updating tasks yielded a significant moderate effect size, $g=0.56$ (0.19), $p<0.01$ (see Fig. 2B). Chi-square test for heterogeneity was not significant, $I^{2}=0 \%, Q(9)=$ $6.1827, p=0.72$. Analysis of unilateral a-tDCS effects on set shifting tasks did not yield a significant effect size, $g=-0.04$ (0.32), $p=$ 0.5520 . Chi-square test for heterogeneity was significant, $I^{2}=69.92 \%$, $Q(12)=39.89, p<0.0001$. Analysis of unilateral a-tDCS effects on inhibition tasks did not yield a significant effect size, $g=-0.10(0.18)$, $p=0.55$. Chi-square test for heterogeneity was not significant, $I^{2}=$ 


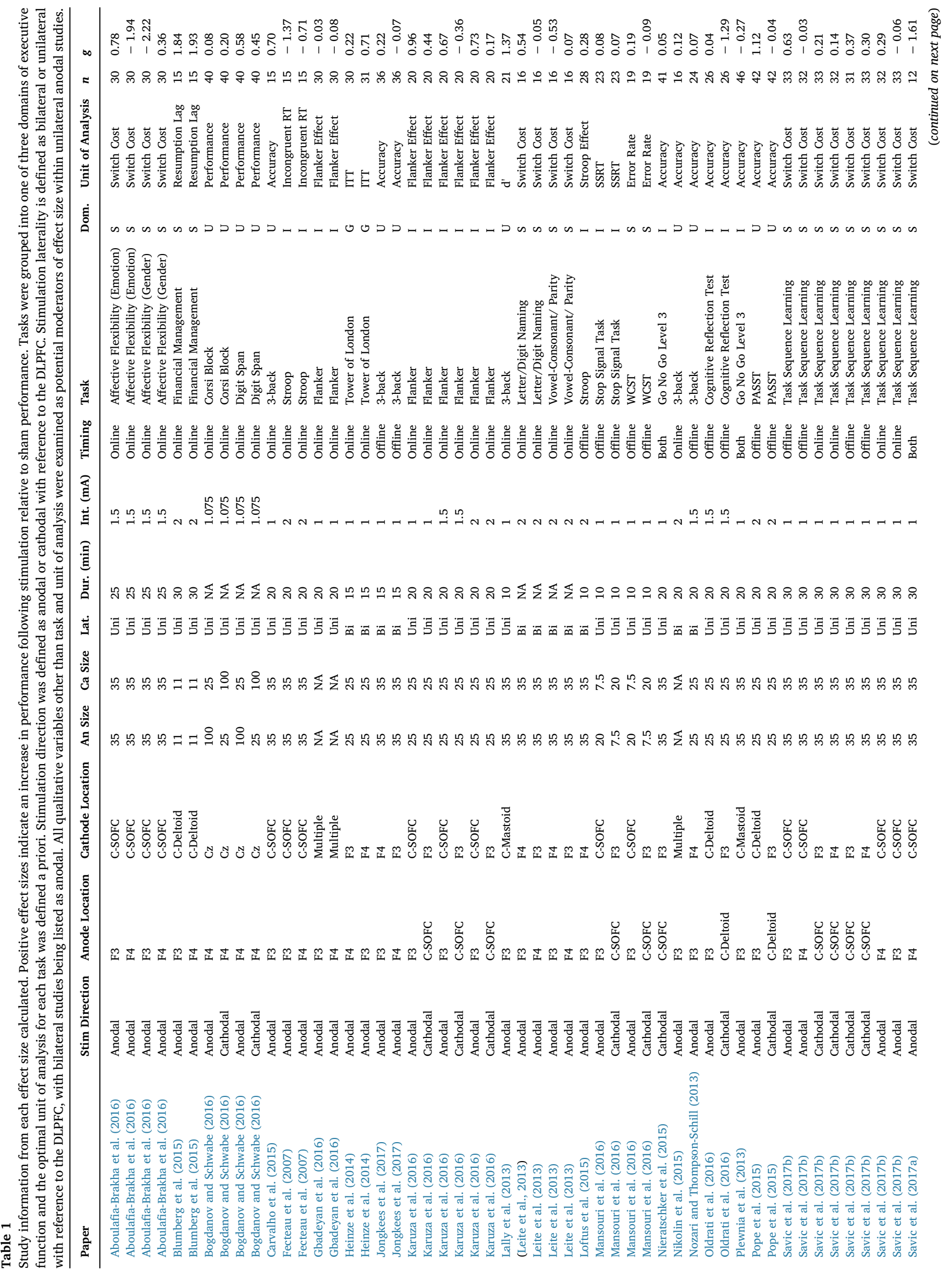




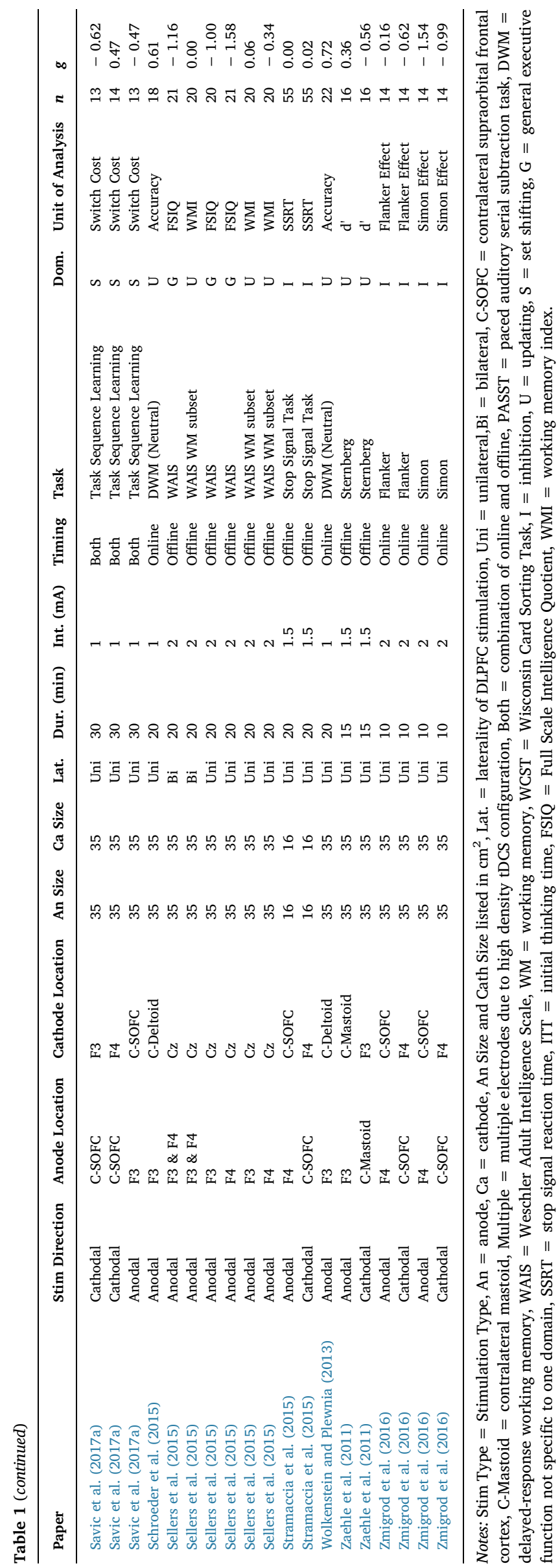



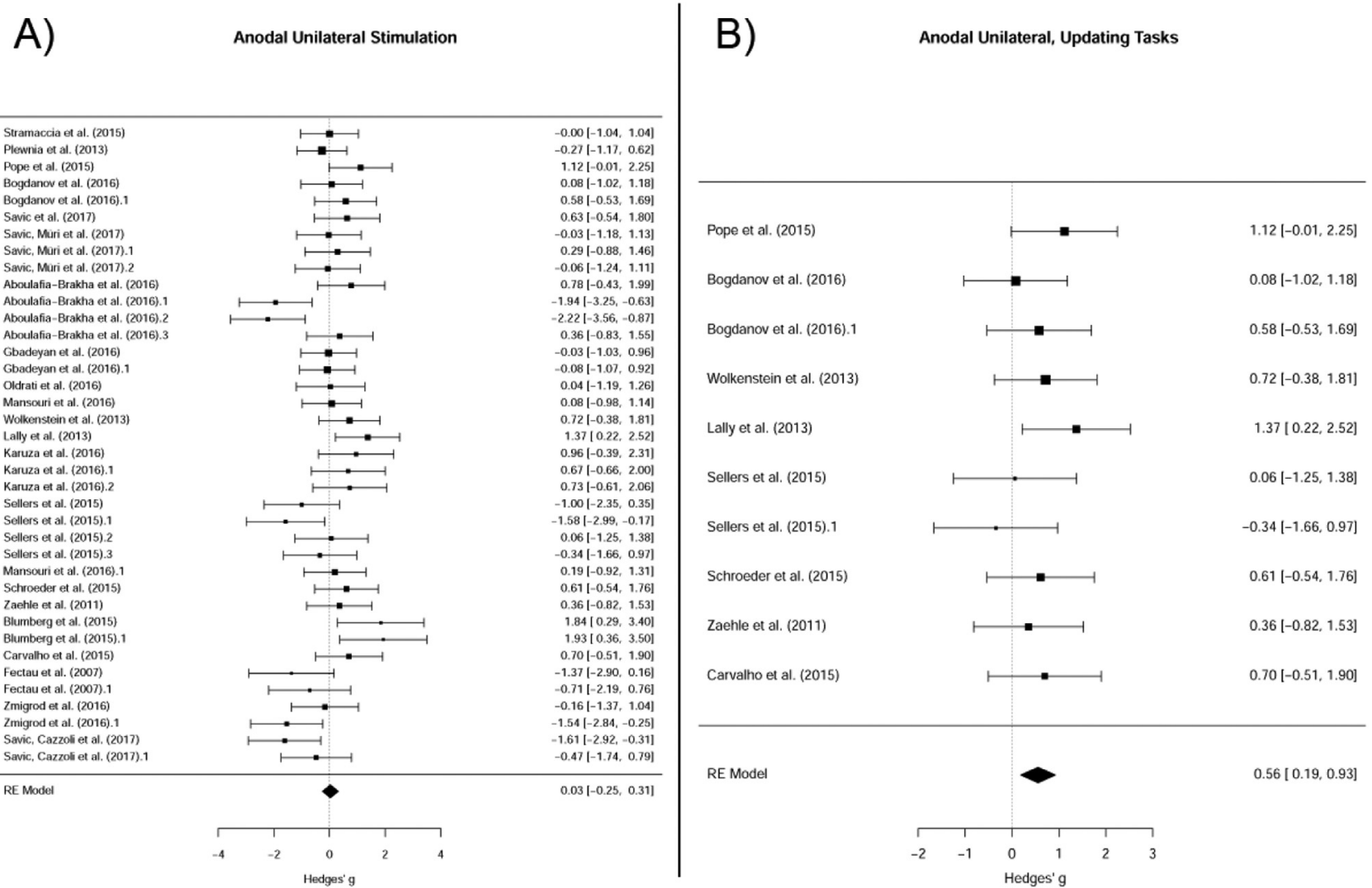

Fig. 2. A) Effect of anodal stimulation on global EF (all tasks). No significant effect was found. B) Effect of anodal stimulation on updating task subgroup. Significant improvement was found compared to sham stimulation performance.

$11.49 \%, Q(12)=13.55, p=0.33$. Thus, analyses suggest that unilateral a-tDCS had a moderately large effect on updating tasks, but not on inhibition or set shifting tasks. Although analysis of set shifting tasks revealed significant heterogeneity, the small sample of effect sizes prevented further subgroup analysis based on other possible moderators.

\subsection{Exploratory analyses}

The first planned moderator analysis conducted examined the effect anode size. The meta-regression revealed a significant effect, $Q M(1)=$ $5.99, p=0.01$, indicating larger effect sizes in studies using smaller anodes. The test of residual heterogeneity was significant, $Q E(34)=$ $67.1, p<0.001$, indicating a significant proportion of heterogeneity of effect scores not accounted for by anode size. For the subgroup analysis, electrode size was split on the mean, resulting in one group with anodes sized $35 \mathrm{~cm}^{2}(N=19)$ and one group with anodes sized $25 \mathrm{~cm}^{2}$ or smaller $(N=11)$. Further division of these groups was not undertaken due to the relatively small number of effect sizes from studies using anodes smaller than $35 \mathrm{~cm}^{2}$. The large anode group yielded a nonsignificant effect, $g=-0.26$ (0.19), $p=0.19$, while the small anode group yielded a significant moderate effect, $g=0.55(0.18), p=0.002$ (see Fig. 3).

Anode location (F3 vs. F4) was dummy coded and assessed as a potential moderator with meta-regression. Anode location was a trending moderator, $Q M(1)=3.6, p=0.06$. However, subgroup analyses yielded nonsignificant effect sizes from both the F3 and F4 groups, $p=0.14$ and $p=0.21$ respectively. Although the F3 and F4 groups were significantly different from each other, neither group yielded a total effect size significantly different from zero.

A moderator analysis was then conducted examining the effect of cathode location. All studies examined placed the cathode on either the midpoint of the head ( $\mathrm{Cz}, N=6$ ), the contralateral supraorbital cortex $(N=21)$, the contralateral mastoid $(N=3)$, or the contralateral deltoid $(N=6)$. Studies that used multiple reference electrodes were excluded from these analyses. $\mathrm{Cz}$ and the supraorbital cortex were grouped together into a cranial group while the mastoid and deltoid were grouped together into an extracranial group. Cathode location was dummy coded such that the cranial group was the reference group. The meta-regression yielded a significant result, $Q M(1)=9.01, p=0.003$, indicating larger effect sizes resulting from studies using extracranial cathodes. The test of residual heterogeneity was significant, $Q E(34)=$ $62.0603, p=0.002$. A subgroup analysis revealed a nonsignificant effect from studies using cranial cathodes, $g=-0.21$ (0.17), $p=0.21$, however, studies using extracranial cathodes yielded a moderate to large effect, $g=0.74$ (0.25), $p=0.003$ (see Fig. 4).

Stimulation intensity was not a significant moderator of unilateral atDCS, $p=0.31$. Following Dedoncker et al. (2016), we then examined current density as a moderator, calculating current density as anode size divided by current intensity. Current density was associated with effect size at a trend-level, $Q M(1)=3.44, p=0.06$. The test of residual heterogeneity was significant, $Q E(34)=62.0603, p=0.0023$. Current density was then divided into high-density, medium-density and lowdensity groups. High-density was defined as greater than or equal to the third quartile $\left(0.0579 \mathrm{~mA} / \mathrm{cm}^{2}\right)$ while low-density was less than or equal to the first quartile $\left(0.0286 \mathrm{~mA} / \mathrm{cm}^{2}\right)$. Subgroup analyses revealed a moderate to large positive effect of high-density stimulation, $g$ $=0.6(0.23), p=0.01$, a moderate negative effect of medium-density stimulation, $g=-0.52(0.25), p=0.04$, and a nonsignificant effect of low density stimulation, $p=0.27$. Density charge was calculated as the product of current density and stimulation duration. Density charge was calculated only for offline studies because online designs often lasted for a variable amount of time depending on how long a participant took to complete a task. Density charge was not a significant 


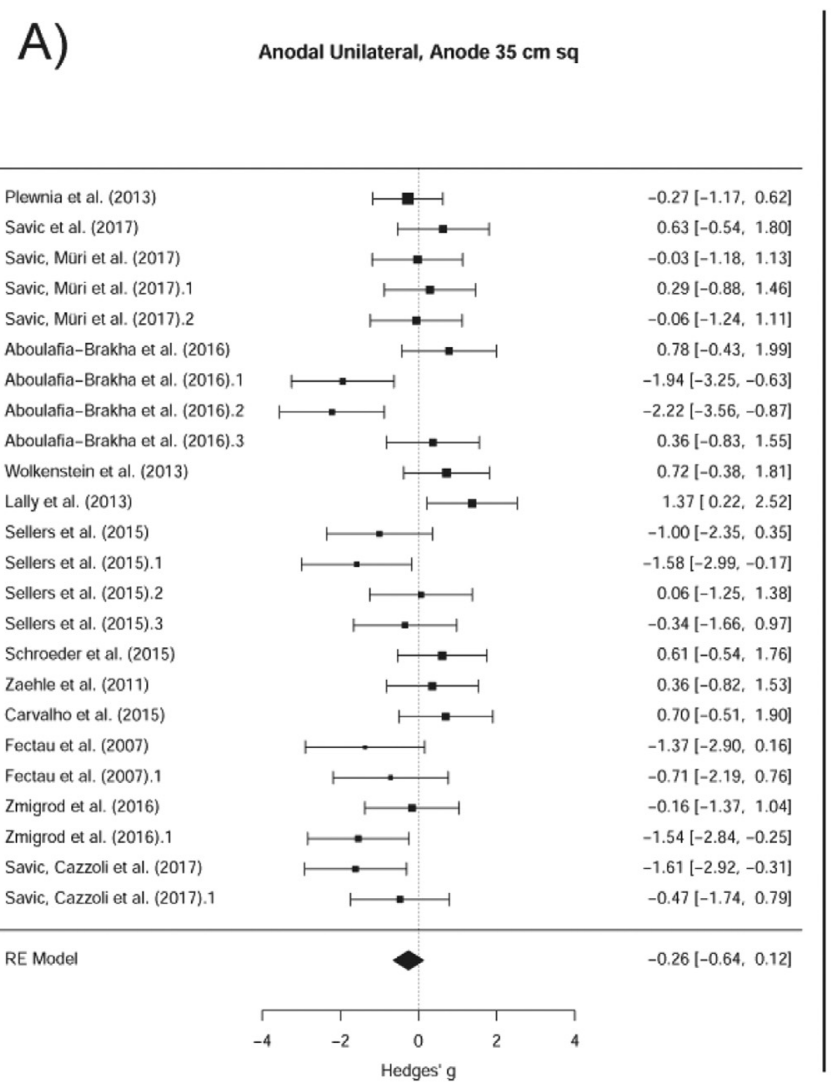

B)

Anodal Unilateral, Anode $25 \mathrm{~cm}$ sq or less

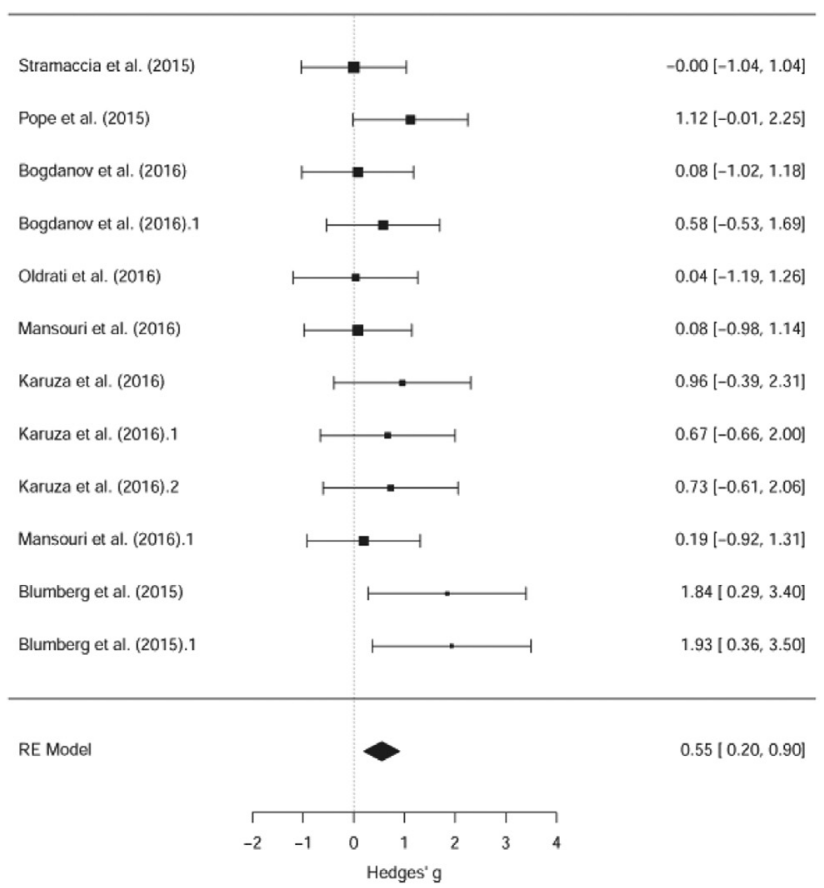

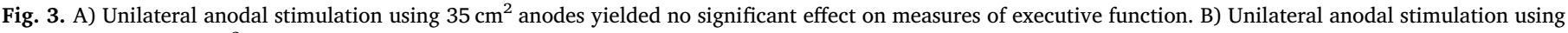
anodes that were $25 \mathrm{~cm}^{2}$ or smaller resulted in a significant increase in performance on measures of executive function relative to sham stimulation.

moderator, $p=0.77$.

\section{Discussion}

The current work aims to quantify the effectiveness of DLPFC tDCS in modulating executive function in healthy populations. In order to further understand the mechanism of action, the effect of a-tDCS on inhibition, updating, and set-shifting were examined independently. The effects of stimulation parameters and electrode montage on tDCS effectiveness were examined to address the variation in stimulation parameters and electrode placement across studies. While no significant effect of stimulation was found on executive function in general, unilateral a-tDCS significantly improved performance on updating measures. Additionally, unilateral a-tDCS had a greater effect on executive function when small anodes or extracranial cathodes were used. Finally, high current density was associated with a greater positive effect on executive function than medium or low current density.

\subsection{Anodal stimulation effects on executive function}

The current work suggests there is no significant effect of singlesession DLPFC tDCS on global executive function, in line with recent meta-analyses examining general cognition and tDCS (Dedoncker et al., 2016; Horvath et al., 2015). However, when domains of executive function were analyzed individually, a significant effect of unilateral atDCS on working memory performance emerged, while no significant effect was found on set shifting ability nor inhibition. The finding is in contrast to a previous analysis that found no effect of single-session tDCS on working memory (Horvath et al., 2015). The difference may be attributable to the fact that the current analysis was constrained to unilateral stimulation of the DLPFC. Another analysis that included working memory training paradigms (rather than solely single-session
tDCS) found evidence of a significant effect of tDCS on working memory, although the effect was no longer significant after correction for publication bias (Lewis and Bates, 2013). A trim and fill analysis of our updating group revealed zero estimated missing studies, indicating publication bias was unlikely in our subgroup analysis.

It is possible that the DLPFC is more integral to working memory than to set shifting and inhibition, explaining the significant effect on the updating domain and null effects on the other two. Indeed, computational neuroscience models have suggested that shifting and inhibition are largely dependent on dynamic gating of the contents of working memory, mediated by dopaminergic pathways between PFC and basal ganglia (Hazy et al., 2007; O'Reilly, 2002). While neuroimaging meta-analyses have found consistent significant activation of the DLPFC during working memory tasks (Curtis and D'Esposito, 2003; Wager et al., 2004), the DLPFC was not found to be significantly active for task-switching paradigms unless the significance threshold was raised to 0.13 (Wager et al., 2004). Instead, the most consistently active areas during task switches were in the parietal region, an area likely untouched by the majority of the electrode montages in the current analysis. A more recent meta-analysis of task switching fMRI studies found that the inferior frontal junction (but not DLPFC) and posterior parietal cortex were consistently activated across switching types (Kim et al., 2012). Work on inhibition has focused on a role for the right PFC, and specifically the right ventrolateral PFC (Aron et al., 2014, 2004; Chatham et al., 2012; Munakata et al., 2011). This previous work has focused on classical inhibition tasks such as go/no-go and stop-signal, while most of the papers included for inhibition in the current analysis used the Flanker and Stroop tasks. While the DLPFC is thought to be important for executive function during Stroop and Flanker tasks, a number of other regions are also consistently active, namely the anterior cingulate cortex (Nee et al., 2007). It is possible that the anterior cingulate is central or at least critical to the performance of these tasks, 


\begin{tabular}{l} 
Stramaccia et al. (2015) \\
Bogdanov et al. (2016) \\
Bogdanov et al. (2016).1 \\
Savic et al. (2017) \\
Savic, Müri et al. (2017) \\
Savic, Müri et al. (2017).1 \\
Savic, Muri et al. (2017).2 \\
Aboulafia-Brakha et al. (2016) \\
Aboulafia-Brakha et al. (2016).1 \\
Aboulafia-Brakha et al. (2016).2 \\
Aboulafia-Brakha et al. (2016).3 \\
Mansouri et al. (2016) \\
Karuza et al. (2016) \\
Karuza et al. (2016).1 \\
Karuza et al. (2016).2 \\
Sellers et al. (2015) \\
Sellers et al. (2015).1 \\
Sellers et al. (2015).2 \\
Sellers et al. (2015).3 \\
Mansouri et al. (2016).1 \\
Carvalho et al. (2015) \\
Fectau et al. (2007) \\
Fectau et al. (2007).1 \\
Zmigrod et al. (2016) \\
Zmigrod et al. (2016).1 \\
Savic, Cazzoli et al. (2017) \\
Savic, Cazzoli et al. (2017).1 \\
\hline RE Model
\end{tabular}

Couphe et al. (2011)

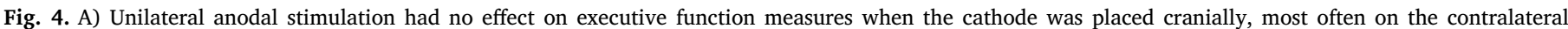

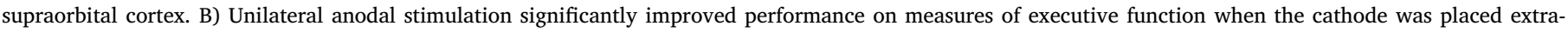
cranially, most often on the contralateral deltoid.

such that DLPFC stimulation alone is not effective. Thus, while the link between the DLPFC and updating is well supported, more work is necessary to establish the importance of the DLPFC (possibly in conjunction with other prefrontal regions) to shifting and inhibition.

The significant effect of stimulation on updating paradigms might also be explained by the effects of a-tDCS on GABA. The unity diversity model defines updating as "updating and monitoring of working memory representations" and "closely linked to the notion of working memory" (Miyake et al., 2000). The authors note that this domain specifically is often associated with DLPFC function (Miyake et al., 2000). A-tDCS has been found to result in a decrease in GABA concentration in the motor cortex, (Antonenko et al., 2017; Bachtiar et al., 2015; Kim et al., 2014; Stagg and Nitsche, 2011; Tremblay et al., 2013) although this finding was not replicated in one sample (Amadi et al., 2015) and may depend on age (Heise et al., 2014). Decreases in DLPFC GABA are associated with higher accuracy rates in working memory tasks (Michels et al., 2012; Yoon et al., 2016), providing a possible neural mechanism for updating improvement.

The lack of effects on task-switching and inhibition is somewhat surprising, given the link between task switching and the DLPFC (Kim et al., 2012; Wager et al., 2005). GABA regulation might also underlie the lack of effects on inhibition and set-shifting tasks; while reductions in GABA are associated with updating improvements in healthy individuals, little is known about the relationship between DLPFC GABA and the other two domains. One study found that prefrontal GABA reductions can be detrimental to set-shifting ability (Enomoto et al., 2011) and literature search revealed no studies demonstrating a relationship between GABA in the lateral PFC and inhibition paradigms.

Neurotransmitter changes after stimulation may also underlie the lack of significant effect of c-tDCS. While a-tDCS decreases GABA in the motor cortex, c-tDCS primarily decreases glutamate. However, reductions in glutamate may induce secondary reductions in GABA (Stagg et al., 2013). Reductions of both excitatory and inhibitory neurotransmitters may effectively cancel each other out. However, neurotransmitters alone do not address the absence of online c-tDCS effects. More investigation is necessary to determine why c-tDCS may not be effective in modulating executive function.

\subsection{Methodological moderators}

Unilateral a-tDCS utilizing $25 \mathrm{~cm}^{2}$ anodes or smaller was found to significantly enhance executive function, while $35 \mathrm{~cm}^{2}$ anodes did not. The greater effectiveness of smaller electrodes is likely due to increased focality of stimulation - larger electrodes are more likely to affect neighboring areas yielding incidental and unintended effects on nontarget sites. The analysis was not able to replicate findings from a previous meta-analysis regarding the significance of current density or density charge as moderators of a-tDCS effect, although current density was a trending moderator (Dedoncker et al., 2016). The difference may be due to the metrics analyzed; the previous analysis focused on accuracy and reaction time separately rather than identifying the most relevant metric for a given task. Additionally, the current analysis constrained density-charge analysis to offline studies due to variability in the duration in online studies within samples, unlike Dedoncker et al. The current work supports the idea that smaller electrodes yield more consistent results due to more focal stimulation, but not due to increased current density (further refuted by the lack of moderating effects of stimulation intensity in general).

Single-session unilateral a-tDCS was found to enhance executive function when an extracranial cathode was used, whereas cranial cathodes led to no effect. The finding is in contrast to a previous analysis that found no significant effect of reference electrode location, although the authors do suggest they expected extracranial reference electrodes to lead to stronger effects (Dedoncker et al., 2016). When the 


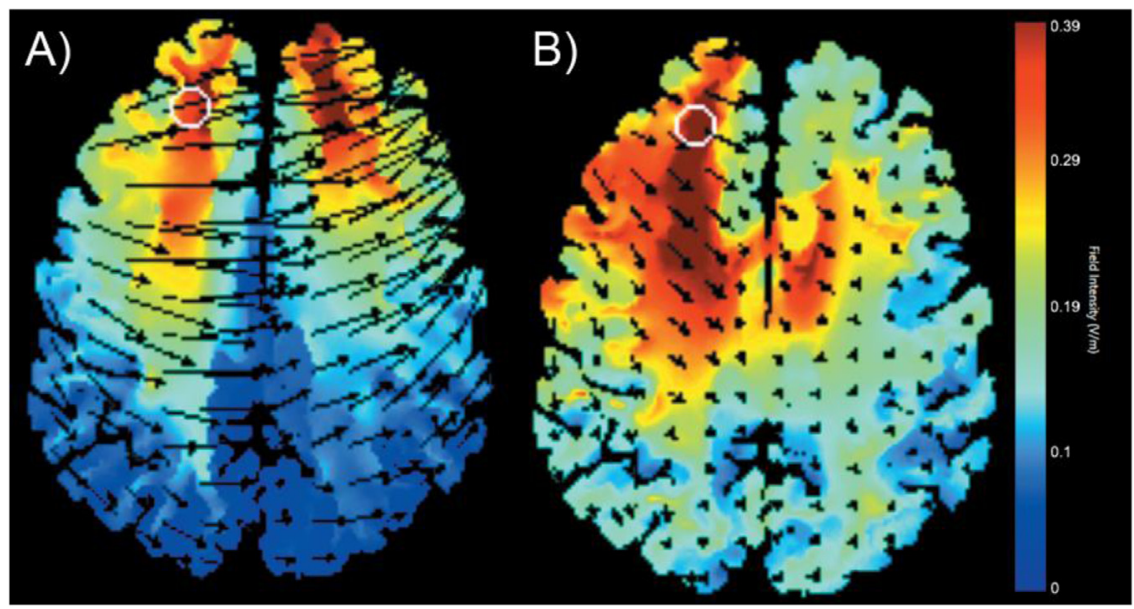

Fig. 5. Current intensity simulations depicting anodal stimulation targeting the left DLPFC. Current models were obtained using Soterix hdTargets software simulating $2 \mathrm{~mA}$ stimulation with $35 \mathrm{~cm}^{2}$ sponge electrodes (anode at F3). Arrows indicate stimulation direction, color indicates stimulation intensity, circle indicates F3 target site. A) Concentrated, high intensity stimulation of the right DLPFC when a cranial cathode over the supraorbital Fp2 site is used likely inhibits function in the right DLPFC. B) Diffuse, low intensity stimulation outside of the left DLPFC when an extracranial cathode is used (right neck sites) decreases the likelihood of unintended inhibition in prefrontal areas.

cathode is placed on either the midpoint of the head or the supraorbital cortex, current is diffused through potentially task relevant areas causing unwanted inhibition of these areas, as simulated in Soterix HDExplore software (Datta et al., 2013; Huang et al., 2017; see Fig. 5) Extracranial cathodes result in less inhibition in the frontal cortex, likely increasing facilitation of executive function relative to cranial cathodes. The nonsignificant effect of bilateral tDCS may be similarly explained by concurrent excitation and inhibition of task-relevant areas.

Differences in findings between the current study and previous analyses may be due to an increase in studies published recently that fit our criteria, allowing for greater statistical power in our analyses. Additionally, the current study focused on the DLPFC and executive function, whereas previous studies were only able to focus on either the DLPFC or executive function due to relatively small sample sizes (Dedoncker et al., 2016; Horvath et al., 2015). The inclusion of brain areas not centrally related to executive function may have diluted effect sizes with respect to moderators and a-tDCS effects on updating tasks. Inclusion of any cognitive task in relation to DLPFC function may have had a similar effect. For example, long term memory tasks fall under the umbrella of cognitive function but are not directly related to the DLPFC (Blumenfeld and Ranganath, 2007). Finally, the current study considered bilateral tDCS separately from unilateral a-tDCS to account for the consistent inhibition of one DLPFC and excitation of the other, whereas previous analyses did not.

\subsection{Implications for clinical work}

Modulation of updating ability may account for some of the improvements seen in disordered populations. Ruminative thinking contributes to the maintenance of MDD and has been linked to working memory deficits that are consistently demonstrated by individuals with MDD (De Lissnyder et al., 2012; Joormann et al., 2011; NolenHoeksema et al., 2008). One pilot study, although confirming the relationship between working memory and rumination in MDD, did not find a significant effect of bilateral DLPFC tDCS in affecting either (Vanderhasselt et al., 2015). However, the authors note that the inhibition of the right DLPFC due to the bilateral montage may be the cause of the lack of findings, leaving open the possibility that unilateral a-tDCS may improve depressive symptoms by improving ruminative behavior.

Schizophrenia has also been associated with deficits in working memory (Lee and Park, 2005). Furthermore, fMRI analysis identified DLPFC dysfunction as a possible source of working memory dysfunction in schizophrenia (Tan et al., 2005), supporting the idea that anodal stimulation of the DLPFC ameliorates working memory deficits in schizophrenia. A correlational analysis found that working memory ability is related to a number of other cognitive functions only in people with schizophrenia (Silver et al., 2003). The authors concluded from these correlational analyses that working memory is a core symptom of schizophrenia, underlying many other deficits. Therefore, it is possible that improvements in working memory caused by DLPFC stimulation might drive broader cognitive improvements seen in people with schizophrenia following stimulation (Brunelin et al., 2012) and not in healthy controls.

\section{Limitations}

There are several issues that the current study was not able to address due to the relatively small sample size. Further analysis examining the effect of each bilateral configuration (anode over F3 and cathode over F4 or vice versa) with respect to the task performed is likely necessary to fully examine the effects of bilateral stimulation. Exciting the left DLPFC and inhibiting the right may be beneficial for some tasks and detrimental to others. However, the current analysis lacks the power necessary to examine task laterality as a moderator. Additionally, findings related to current density seem counterintuitive. High density stimulation yielded a significant positive effect, medium density a significant negative effect and low density no effect. The relationship between current density and effect size may be clarified by examining additional moderators such as electrode size. However, the current study lacks the statistical power to conduct these analyses. Furthermore, individual differences in response to stimulation may play a role in some of the null effects found in the current analysis. However, meta-analyses are capable only of examining inter-study variation, not intra-study variation (Gurevitch et al., 2018). Finally, as more data becomes available it may be beneficial to analyze the effect of tDCS on individual tasks rather than simply domains, as the effects might not be homogeneous within domains.

\section{Conclusions}

The current work is a step towards understanding how single-session DLPFC tDCS effects executive function in healthy populations. Quantifying the effects of DLPFC stimulation on domains of executive function separately will help clarify the degree to which the DLPFC is involved in each domain. Additionally, greater understanding of the behavioral effects of the stimulation may help us understand how and why tDCS may be used to treat disorders related to executive function such as MDD and schizophrenia. Analyses examining the effects of methodological influences on tDCS effect may help determine which electrode configurations (specifically with respect to reference electrode placement) and design considerations may be most effective in future work. The current work suggests that anodal tDCS of the DLPFC 
is more effective in modulating information updating than inhibition or set-shifting ability. Additionally, the current work exemplifies the importance of study design, as anodal stimulation of the DLPFC only significantly affected general executive function when extracranial cathodes or small anodes were used.

\section{Funding}

This research did not receive any specific grant from funding agencies in the public, commercial, or not-for-profit sectors.

\section{References}

Aboulafia-Brakha, T., Manuel, A.L., Ptak, R., 2016. Prefrontal transcranial direct current stimulation facilitates affective flexibility. Neuropsychologia $86,13-18$. http://dx. doi.org/10.1016/j.neuropsychologia.2016.03.030.

Amadi, U., Allman, C., Johansen-Berg, H., Stagg, C.J., 2015. The homeostatic interaction between anodal transcranial direct current stimulation and motor learning in humans is related to GABA $<$ inf $>\mathrm{A}</$ inf $>$ activity. Brain Stimul. 8, 898-905. http://dx. doi.org/10.1016/j.brs.2015.04.010.

Antonenko, D., Schubert, F., Bohm, F., Ittermann, B., Aydin, S., Hayek, D., Grittner, U., Flöel, A., 2017. tDCS-Induced Modulation of GABA Levels and Resting-State Functional Connectivity in Older Adults. J. Neurosci. 37, 4065-4073. http://dx.doi. org/10.1523/JNEUROSCI.0079-17.2017.

Aron, A.R., Robbins, T.W., Poldrack, R.A., 2014. Inhibition and the right inferior frontal cortex: one decade on. Trends Cogn. Sci. 18, 177-185. http://dx.doi.org/10.1016/j. tics.2013.12.003.

Aron, A.R., Robbins, T.W., Poldrack, R.A., 2004. Inhibition and the right inferior frontal cortex. Trends Cogn. Sci. 8, 170-177. http://dx.doi.org/10.1016/J.TICS.2004.02 010 .

Bachtiar, V., Near, J., Johansen-Berg, H., Stagg, C.J., 2015. Modulation of GABA and resting state functional connectivity by transcranial direct current stimulation. Elife 4, e08789. http://dx.doi.org/10.7554/eLife.08789.

Berlim, M.T., Van Den Eynde, F., Daskalakis, Z.J., 2013. Clinical utility of transcranial direct current stimulation (tDCS) for treating major depression: a systematic review and meta-analysis of randomized, double-blind and sham-controlled trials. J. Psychiatr. Res. 47, 1-7. http://dx.doi.org/10.1016/j.jpsychires.2012.09.025.

Biringer, E., Lundervold, A., Stordal, K., Mykletun, A., Egeland, J., Bottlender, R., Lund, A., 2005. Executive function improvement upon remission of recurrent unipolar depression. Eur. Arch. Psychiatry Clin. Neurosci. 255, 373-380. http://dx.doi.org/ 10.1007/s00406-005-0577-7.

Blumberg, E.J., Foroughi, C.K., Scheldrup, M.R., Peterson, M.S., Boehm-Davis, D.A., Parasuraman, R., 2015. Reducing the disruptive effects of interruptions with noninvasive brain stimulation. Hum. Factors 57, 1051-1062. http://dx.doi.org/10.1177/ 0018720814565189.

Blumenfeld, R.S., Ranganath, C., 2007. Prefrontal cortex and long-term memory encoding: an integrative review of findings from neuropsychology and neuroimaging. Neuroscientist 13, 280-291. http://dx.doi.org/10.1177/1073858407299290.

Bogdanov, M., Schwabe, L., 2016. Transcranial stimulation of the dorsolateral prefrontal cortex prevents stress-induced working memory deficits. J. Neurosci. 36, 1429-1437. http://dx.doi.org/10.1523/JNEUROSCI.3687-15.2016.

Boggio, P.S., Sultani, N., Fecteau, S., Merabet, L., Mecca, T., Pascual-Leone, A., Basaglia, A., Fregni, F., 2008. Prefrontal cortex modulation using transcranial DC stimulation reduces alcohol craving: a double-blind, sham-controlled study. Drug Alcohol Depend. 92, 55-60. http://dx.doi.org/10.1016/j.drugalcdep.2007.06.011.

Brunelin, J., Mondino, M., Gassab, L., Haesebaert, F., Gaha, L., Suaud-Chagny, M.-F., Saoud, M., Mechri, A., Poulet, E., 2012. Examining Transcranial Direct-Current Stimulation (tDCS) as a Treatment for hallucinations in schizophrenia. Am. J. Psychiatry 169, 719-724. http://dx.doi.org/10.1176/appi.ajp.2012.11071091.

Brunoni, A.R., Ferrucci, R., Bortolomasi, M., Vergari, M., Tadini, L., Boggio, P.S., Giacopuzzi, M., Barbieri, S., Priori, A., 2011. Transcranial direct current stimulation (tDCS) in unipolar vs. bipolar depressive disorder. Neuro-Psychopharmacol. Biol. Psychiatry 35, 96-101. http://dx.doi.org/10.1016/j.pnpbp.2010.09.010.

Carvalho, S., Boggio, P.S., Gonçalves, Ó.F., Vigário, A.R., Faria, M., Silva, S., Gaudencio Do Rego, G., Fregni, F., Leite, J., 2015. Transcranial direct current stimulation based metaplasticity protocols in working memory. Brain Stimul. 8, 289-294. http://dx. doi.org/10.1016/j.brs.2014.11.011.

Chatham, C.H., Claus, E.D., Kim, A., Curran, T., Banich, M.T., Munakata, Y., 2012. Cognitive control reflects context monitoring, not motoric stopping, in response in hibition. PLoS One 7, e31546. http://dx.doi.org/10.1371/journal.pone.0031546.

Cools, R., D'Esposito, M., 2011. Inverted-U-shaped dopamine actions on human working memory and cognitive control. Biol. Psychiatry 69. http://dx.doi.org/10.1016/j. biopsych.2011.03.028.

Curtis, C.E., D'Esposito, M., 2003. Persistent activity in the prefrontal cortex during working memory. Trends Cogn. Sci. 7, 415-423. http://dx.doi.org/10.1016/S13646613(03)00197-9.

Datta, A., Zhou, X., Su, Y., Parra, L.C., Bikson, M., 2013. Validation of finite element model of transcranial electrical stimulation using scalp potentials: implications for clinical dose. J. Neural Eng. 10, 36018. http://dx.doi.org/10.1088/1741-2560/10/ $3 / 036018$.

De Lissnyder, E., Koster, E.H.W., De Raedt, R., 2012. Emotional Interference in Working Memory is Related to Rumination. Cogn. Ther. Res. 36, 348-357. http://dx.doi.org/ 10.1007/s10608-011-9352-4.

Dedoncker, J., Brunoni, A.R., Baeken, C., Vanderhasselt, M.-A., 2016. A systematic review and meta-analysis of the effects of Transcranial Direct Current Stimulation (tDCS) over the dorsolateral prefrontal cortex in healthy and neuropsychiatric samples: influence of stimulation parameters. Brain Stimul. 9, 1-17. http://dx.doi.org/10.1016/ j.brs.2016.04.006.

Enomoto, T., Tse, M.T., Floresco, S.B., 2011. Reducing prefrontal Gamma-aminobutyric acid activity induces cognitive, behavioral, and dopaminergic abnormalities that resemble schizophrenia. Biol. Psychiatry 69, 432-441. http://dx.doi.org/10.1016/j. biopsych.2010.09.038.

Fecteau, S., Knoch, D., Fregni, F., Sultani, N., Boggio, P., Pascual-Leone, A., 2007. Diminishing risk-taking Behavior by modulating activity in the prefrontal cortex: a direct current stimulation study. J. Neurosci. 27, 12500-12505. http://dx.doi.org/ 10.1523/JNEUROSCI.3283-07.2007.

Fossati, P., Ergis, A.M., Allilaire, J.F., 2002. Executive functioning in unipolar depression: a review. Encephale 28, 97-107.

Fregni, F., Boggio, P.S., Nitsche, M., Marcolin, M. a., Rigonatti, S.P., Pascual-Leone, A., 2006. Treatment of major depression with transcranial direct current stimulation. Bipolar Disord. 8, 203-205. http://dx.doi.org/10.1111/j.1399-5618.2006.00291.x.

Gbadeyan, O., McMahon, K., Steinhauser, M., Meinzer, M., 2016. Stimulation of dorsolateral prefrontal cortex enhances adaptive cognitive control: a high-definition transcranial direct current stimulation study. J. Neurosci. 36, 12530-12536. http:// dx.doi.org/10.1523/JNEUROSCI.2450-16.2016.

Gill, J., Shah-Basak, P.P., Hamilton, R., 2015. It's the thought that counts: examining the task-dependent effects of transcranial direct current stimulation on executive function. Brain Stimulation 8, 253-259. http://dx.doi.org/10.1016/j.brs.2014.10.018.

Gurevitch, J., Koricheva, J., Nakagawa, S., Stewart, G., 2018. Meta-analysis and the science of research synthesis. Nature 555, 175-182. http://dx.doi.org/10.1038/ nature25753.

Hazy, T.E., Frank, M.J., O'Reilly, R.C., 2007. Towards an executive without a homunculus: computational models of the prefrontal cortex/basal ganglia system. Philos. Trans. R. Soc. B Biol. Sci. 362, 1601-1613. http://dx.doi.org/10.1098/rstb. 2007.2055.

Hedges, L.V., 1981. Distribution theory for Glass's estimator of effect size and related estimators. J. Educ. Stat. 6, 107-128.

Heinze, K., Ruh, N., Nitschke, K., Reis, J., Fritsch, B., Unterrainer, J.M., Rahm, B., Weiller, C., Kaller, C.P., 2014. Transcranial direct current stimulation over left and right DLPFC: lateralized effects on planning performance and related eye movements. Biol. Psychol. 102, 130-140. http://dx.doi.org/10.1016/j.biopsycho.2014.07.019.

Heise, K.-F., Niehoff, M., Feldheim, J.-F., Liuzzi, G., Gerloff, C., Hummel, F.C., 2014. Differential behavioral and physiological effects of anodal transcranial direct current stimulation in healthy adults of younger and older age. Front. Aging Neurosci. 6, 146. http://dx.doi.org/10.3389/fnagi.2014.00146.

Horvath, J.C., Forte, J.D., Carter, O., 2015. Quantitative review finds no evidence of cognitive effects in healthy populations from single-session transcranial direct current stimulation (tDCS). Brain Stimul. 8, 535-550. http://dx.doi.org/10.1016/j.brs.2015. 01.400 .

Huang, Y., Liu, A.A., Lafon, B., Friedman, D., Dayan, M., Wang, X., Bikson, M., Doyle, W.K., Devinsky, O., Parra, L.C., 2017. Measurements and models of electric fields in the in vivo human brain during transcranial electric stimulation. Elife 6, 1-26. http:// dx.doi.org/10.7554/eLife.18834.

Imburgio M., Code and Coding Sheet and Coding Manual for Analyses, Published: 3 May 2018, Version 1, http://dx.doi.org/10.17632/j6nr62yzs3.1, https://data.mendeley. $\mathrm{com} /$ datasets/j6nr62yzs3/1.

Jongkees, B.J., Sellaro, R., Beste, C., Nitsche, M.A., Kühn, S., Colzato, L.S., 2017. LTyrosine administration modulates the effect of transcranial direct current stimulation on working memory in healthy humans. Cortex 90, 103-114. http://dx.doi.org/ 10.1016/j.cortex.2017.02.014.

Joormann, J., Levens, S.M., Gotlib, I.H., 2011. Sticky thoughts. Psychol. Sci. 22, 979-983. http://dx.doi.org/10.1177/0956797611415539.

Kalu, U.G., Sexton, C.E., Loo, C.K., Ebmeier, K.P., 2012. Transcranial direct current stimulation in the treatment of major depression: a meta-analysis. Psychol. Med. 42, 1791-1800. http://dx.doi.org/10.1017/S0033291711003059.

Karuza, E.A., Balewski, Z.Z., Hamilton, R.H., Medaglia, J.D., Tardiff, N., Thompson-Schill, S.L., 2016. Mapping the parameter space of tDCS and cognitive control via manipulation of current polarity and intensity. Front. Hum. Neurosci. 10. http://dx.doi. org/10.3389/fnhum.2016.00665.

Kim, C., Cilles, S.E., Johnson, N.F., Gold, B.T., 2012. Domain general and domain preferential brain regions associated with different types of task switching: a MetaAnalysis. Hum. Brain Mapp. 33, 130-142. http://dx.doi.org/10.1002/hbm.21199.

Kim, S., Stephenson, M.C., Morris, P.G., Jackson, S.R., 2014. tDCS-induced alterations in GABA concentration within primary motor cortex predict motor learning and motor memory: a $7 \mathrm{~T}$ magnetic resonance spectroscopy study. NeuroImage 99, 237-243. http://dx.doi.org/10.1016/J.NEUROIMAGE.2014.05.070.

Lally, N., Nord, C.L., Walsh, V., Roiser, J.P., 2013. Does excitatory fronto-extracerebral tDCS lead to improved working memory performance? F1000Research 2, 219. http://dx.doi.org/10.12688/f1000research.2-219.v2.

Lee, J., Park, S., 2005. Working memory impairments in schizophrenia: a meta-analysis. J. Abnorm. Psychol. 114, 599-611 (https://doi.org/2005-15138-012 )(pii) (/r10.1037/0021-843X.114.4.599).

Leite, J., Carvalho, S., Fregni, F., Boggio, P.S.P.S.P.S., Gonçalves, Ó.F.ó.F., Gonçalves, O.F., Gonçalves, Ó.F.Ó.F., 2013. The effects of cross-hemispheric dorsolateral prefrontal cortex transcranial direct current stimulation (tDCS) on task switching. Brain Stimul. 6, 660-667. http://dx.doi.org/10.1016/j.brs.2012.10.006.

Lewis, G.J., Bates, T.C., 2013. Does transcranial direct current stimulation improve healthy working memory?: a meta-analytic review. Psychologist 26, 194-198. http:// 
dx.doi.org/10.1162/jocn.

Li, L.M., Uehara, K., Hanakawa, T., 2015. The contribution of interindividual factors to variability of response in transcranial direct current stimulation studies. Front. Cell. Neurosci. 9, 181. http://dx.doi.org/10.3389/fncel.2015.00181.

Loftus, A.M., Yalcin, O., Baughman, F.D., Vanman, E.J., Hagger, M.S., 2015. The impact of transcranial direct current stimulation on inhibitory control in young adults. Brain Behav. 5. http://dx.doi.org/10.1002/brb3.332.

Mansouri, F.A., Fehring, D.J., Feizpour, A., Gaillard, A., Rosa, M.G.P., Rajan, R., Jaberzadeh, S., 2016. Direct current stimulation of prefrontal cortex modulates errorinduced behavioral adjustments. Eur. J. Neurosci. 44, 1856-1869. http://dx.doi.org/ 10.1111/ejn.13281.

Meltzer, H.Y., McGurk, S.R., 1999. The effects of clozapine, risperidone, and olanzapine on cognitive function in schizophrenia. Schizophr. Bull. 25, 233-255. http://dx.doi. org/10.1093/oxfordjournals.schbul.a033376.

Michels, L., Martin, E., Klaver, P., Edden, R., Zelaya, F., Lythgoe, D., Lüchinger, R., Brandeis, D., O'Gorman, R., 2012. Frontal gaba levels change during working memory. PLoS One 7. http://dx.doi.org/10.1371/journal.pone.0031933.

Miller, E.K., Cohen, J.D., 2001. An integrative theory of prefrontal cortex function. Annu. Rev. Neurosci. 24, 167-202. http://dx.doi.org/10.1146/annurev.neuro.24.1.167.

Minzenberg, M.J., Laird, A.R., Thelen, S., Carter, C.S., Glahn, D.C., 2009. Meta-analysis of 41 functional neuroimaging studies of executive function in schizophrenia. Arch. Gen. Psychiatry 66, 811-822. http://dx.doi.org/10.1001/archgenpsychiatry. 2009.91.

Miyake, A., Friedman, N.P., Emerson, M.J., Witzki, a.H., Howerter, A., Wager, T.D., 2000. The unity and diversity of executive functions and their contributions to complex "Frontal Lobe" tasks: a latent variable analysis. Cogn. Psychol. 41, 49-100. http://dx. doi.org/10.1006/cogp.1999.0734.

Munakata, Y., Herd, S.A., Chatham, C.H., Depue, B.E., Banich, M.T., O’Reilly, R.C., 2011. A unified framework for inhibitory control. Trends Cogn. Sci. http://dx.doi.org/10. 1016/j.tics.2011.07.011.

Nee, D.E., Wager, T.D., Jonides, J.J., 2007. Interference resolution: insights from a metaanalysis of neuroimaging tasks. Cogn. Affect. Behav. Neurosci. 7, 1-17.

Nieratschker, V., Kiefer, C., Giel, K., Krüger, R., Plewnia, C., 2015. The COMT Val/Met polymorphism modulates effects of tDCS on response inhibition. Brain Stimul. 8, 283-288. http://dx.doi.org/10.1016/j.brs.2014.11.009.

Nikolin, S., Loo, C.K., Bai, S., Dokos, S., Martin, D.M., 2015. Focalised stimulation using high definition transcranial direct current stimulation (HD-tDCS) to investigate declarative verbal learning and memory functioning. NeuroImage 117, 11-19. http:// dx.doi.org/10.1016/j.neuroimage.2015.05.019.

Nolen-Hoeksema, S., Wisco, B.E., Lyubomirsky, S., 2008. Rethinking rumination. Perspect. Psychol. Sci. 3, 400-424. http://dx.doi.org/10.1111/j.1745-6924.2008. 00088.x.

Nozari, N., Thompson-Schill, S.L., 2013. More attention when speaking: does it help or does it hurt? Neuropsychologia 51 (3), 2770-2780. http://dx.doi.org/10.1016/j. neuroimage.2015.05.019.

O'Reilly, R.C., 2002. Prefrontal cortex and dynamic categorization tasks: representational organization and neuromodulatory control. Cereb. Cortex 12, 246-257. http://dx. doi.org/10.1093/cercor/12.3.246.

Oldrati, V., Patricelli, J., Colombo, B., Antonietti, A., 2016. The role of dorsolateral prefrontal cortex in inhibition mechanism: a study on cognitive reflection test and similar tasks through neuromodulation. Neuropsychologia 91, 499-508. http://dx. doi.org/10.1016/j.neuropsychologia.2016.09.010.

Plewnia, C., Zwissler, B., Längst, I., Maurer, B., Giel, K., Krüger, R., 2013. Effects of transcranial direct current stimulation (tDCS) on executive functions: influence of COMT Val/Met polymorphism. Cortex 49, 1801-1807. http://dx.doi.org/10.1016/j. cortex.2012.11.002.

Pope, P.A., Brenton, J.W., Miall, R.C., 2015. Task-specific facilitation of cognition by anodal transcranial direct current stimulation of the prefrontal cortex. Cereb. Cortex 25, 4551-4558. http://dx.doi.org/10.1093/cercor/bhv094.

Ptak, R., Schnider, A., 2004. Disorganised memory after right dorsolateral prefrontal damage. Neurocase 10, 52-59. http://dx.doi.org/10.1080/13554790490960495.

R Core Team, 2016. R: A Language and Environment for Statistical Computing.

Robbins, T.W., Arnsten, A.F.T., 2009. The neuropsychopharmacology of fronto-executive function: monoaminergic modulation. Annu. Rev. Neurosci. 32, 267-287. http://dx. doi.org/10.1146/annurev.neuro.051508.135535.

Rogers, M.A., Kasai, K., Koji, M., Fukuda, R., Iwanami, A., Nakagome, K., Fukuda, M., Kato, N., 2004. Executive and prefrontal dysfunction in unipolar depression: a review of neuropsychological and imaging evidence. Neurosci. Res. 50, 1-11. http://dx.doi. org $/ 10.1016 /$ j.neures.2004.05.003.

Rohatgi, A., 2017. WebPlotDigitizer.

Ruf, S.P., Fallgatter, A.J., Plewnia, C., 2017. Augmentation of working memory training by transcranial direct current stimulation (tDCS). Scientific Reports 7 (1), 876. http://dx.doi.org/10.1038/s41598-017.

Savic, B., Cazzoli, D., Müri, R., Meier, B., 2017a. No effects of transcranial DLPFC stimulation on implicit task sequence learning and consolidation. Sci. Rep. 7, 9649. http://dx.doi.org/10.1038/s41598-017-10128-0.

Savic, B., Müri, R., Meier, B., 2017b. A single session of prefrontal cortex transcranial direct current stimulation does not modulate implicit task sequence learning and consolidation. Brain Stimul. 10, 567-575. http://dx.doi.org/10.1016/j.brs.2017.01. 001.

Schroeder, P.A., Ehlis, A.-C., Wolkenstein, L., Fallgatter, A.J., Plewnia, C., 2015. Emotional distraction and bodily reaction: modulation of autonomous responses by anodal tDCS to the Prefrontal cortex. Front. Cell. Neurosci. 9. http://dx.doi.org/10. 3389/fncel.2015.00482.

Sellers, K.K., Mellin, J.M., Lustenberger, C.M., Boyle, M.R., Lee, W.H., Peterchev, A.V., Fröhlich, F., 2015. Transcranial direct current stimulation (tDCS) of frontal cortex decreases performance on the WAIS-IV intelligence test. Behav. Brain Res. 290, 32-44. http://dx.doi.org/10.1016/j.bbr.2015.04.031.

Shiozawa, P., Fregni, F., Benseñor, I.M., Lotufo, P.A., Berlim, M.T., Daskalakis, J.Z. Cordeiro, Q., Brunoni, A.R., 2014. Transcranial direct current stimulation for major depression: an updated systematic review and meta-analysis. Int. J. Neuropsychopharmacol. 17, 1443-1452. http://dx.doi.org/10.1017/ S1461145714000418.

Silver, H., Pablo Feldman, F., Bilker, W., Gur, R.C., 2003. Working memory deficit as a core neuropsychological dysfunction in schizophrenia. Am. J. Psychiatry 16010.

Stagg, C.J., Lin, R.L., Mezue, M., Segerdahl, A., Kong, Y., Xie, J., Tracey, I., 2013. Widespread modulation of cerebral perfusion induced during and after transcranial direct current stimulation applied to the left dorsolateral prefrontal cortex. J. Neurosci. 33, 11425-11431. http://dx.doi.org/10.1523/JNEUROSCI.3887-12.2013.

Stagg, C.J., Nitsche, M.A., 2011. Physiological basis of transcranial direct current stimulation. Neuroscientist 17, 37-53. http://dx.doi.org/10.1177/1073858410386614.

Stramaccia, D.F., Penolazzi, B., Sartori, G., Braga, M., Mondini, S., Galfano, G., 2015. Assessing the effects of tDCS over a delayed response inhibition task by targeting the right inferior frontal gyrus and right dorsolateral prefrontal cortex. Exp. Brain Res. 233, 2283-2290. http://dx.doi.org/10.1007/s00221-015-4297-6.

Tan, H.Y., Choo, W.C., Fones, C.S.L., Chee, M.W.L., 2005. fMRI study of maintenance and manipulation processes within working memory in first-episode schizophrenia. Am. J. Psychiatry 162, 1849-1858. http://dx.doi.org/10.1176/appi.ajp.162.10.1849.

Tremblay, S., Beaulé, V., Lepage, J.-F., Théoret, H., 2013. Anodal transcranial direct current stimulation modulates GABAB-related intracortical inhibition in the M1 of healthy individuals. NeuroReport 24, 46-50. http://dx.doi.org/10.1097/WNR 0b013e32835c36b8.

Tremblay, S., Lepage, J.F., Latulipe-Loiselle, A., Fregni, F., Pascual-Leone, A., Théoret, H., 2014. The uncertain outcome of prefrontal tDCS. Brain Stimul. 7, 773-783. http://dx. doi.org/10.1016/j.brs.2014.10.003.

Vanderhasselt, M.-A., De Raedt, R., Namur, V., Lotufo, P.A., Bensenor, I.M., Boggio, P.S., Brunoni, A.R., 2015. Transcranial electric stimulation and neurocognitive training in clinically depressed patients: a pilot study of the effects on rumination. Prog. NeuroPsychopharmacol. Biol. Psychiatry 57, 93-99. http://dx.doi.org/10.1016/j.pnpbp. 2014.09.015.

Wager, T.D., Jonides, J., Reading, S., 2004. Neuroimaging studies of shifting attention: a meta-analysis. NeuroImage 22, 1679-1693. http://dx.doi.org/10.1016/J. NEUROIMAGE.2004.03.052.

Wager, T.D., Sylvester, C.Y.C., Lacey, S.C., Nee, D.E., Franklin, M., Jonides, J., 2005. Common and unique components of response inhibition revealed by fMRI. NeuroImage 27, 323-340. http://dx.doi.org/10.1016/j.neuroimage.2005.01.054.

Walter, K.H., Palmieri, P.A., Gunstad, J., 2010. More than symptom reduction: changes in executive function over the course of PTSD treatment (n/a-n/a). J. Trauma. Stress 23. http://dx.doi.org/10.1002/jts.20506.

Wolkenstein, L., Plewnia, C., 2013. Amelioration of cognitive control in depression by transcranial direct current stimulation. Biol. Psychiatry 73, 646-651. http://dx.doi. org/10.1016/j.biopsych.2012.10.010.

Yoon, J.H., Grandelis, A., Maddock, R.J., 2016. Dorsolateral prefrontal cortex GABA concentration in humans predicts working memory load processing capacity. J. Neurosci. 36, 11788-11794. http://dx.doi.org/10.1523/JNEUROSCI.1970-16.2016.

Zaehle, T., Sandmann, P., Thorne, J.D., Jäncke, L., Herrmann, C.S., 2011. Transcranial direct current stimulation of the prefrontal cortex modulates working memory performance: combined behavioural and electrophysiological evidence. BMC Neurosci. 12, 2. http://dx.doi.org/10.1186/1471-2202-12-2.

Zmigrod, S., Zmigrod, L., Hommel, B., 2016. Transcranial direct current stimulation (tDCS) over the right dorsolateral prefrontal cortex affects stimulus conflict but not response conflict. Neuroscience 322, 320-325. http://dx.doi.org/10.1016/j. neuroscience.2016.02.046. 